\title{
SECTION 2. EDUCATION
}

\subsection{Formation and development of junior schoolchildren's scientific concepts on the lessons of the integrated course "I explore the world"}

In the modern information society with its socio-economic demands - intensivee growth of high-tech industries and communications, high dynamism of change, globalization processes, rapid growth of knowledge - the direct and main productive force is education and personal intelligence. Only a highly educated person is capable of successful life and further improvement in changing living conditions. That is why the education of the XXI century should become an important resource for the society intellectualization.

The time challenges led to the development of the Concept of the New Ukrainian School [24]. The document emphasizes the learning subjectivity, the need for pupils to master the educational activities methods, the experience of interaction with other people, the formation of the world scientific picture foundations, which is impossible without pupils' development of conceptual apparatus and knowledge, skills and competencies.

Scientific concepts are one of the most important elements of scientific knowledge system, the logical basis of laws and theories. They play an important role in the knowledge system of objects and phenomena of the surrounding world, as they serve as a basis for perception of reality and are a kind of result of its knowledge.

The junior school age deserves special attention in the context of scientific concepts formation. It is at this age that mental operations and actions are formed, moral and intellectual feelings, thinking are actively developed. Characteristic of primary school is the intellectualization of the child's life, meaningfully differentiated process of his personality's individualization. The main task of educating the junior schoolchildren's thinking is to learn new concepts.

The formation and development of ideas and scientific concepts has been and remains the most pressing problem in the junior schoolchildren's education. In the 
context of the research problem modern scientific works are very important. They consider some of its aspects: general issues of scientific concepts formation in junior schoolchildren (T. Barakina), natural ideas and concepts formation in six-year-old first-graders (O. Bida); formation of natural concepts system in the course of studying natural sciences in primary school (O. Varakuta); the role of reference schemes in natural science concepts formation (O. Postnikov); the role of subject lessons in natural science concepts formation (L. Burova); natural science concepts formation and their mental development (T. Bogdanets, M. Sheptukhovsky); didactic conditions for the formation of natural science ideas about the world around us in primary school (M. Matveeva), etc. Scientists emphasize that it is the primary school age that is extremely favorable for the formation and development of scientific concepts.

Long experience in primary education gives confidence to say that junior schoolchildren often learn the concept definition without understanding its essence, operate on terms without realizing the essential features.

Despite the fact that the urgency of this problem is evidenced by modern school practice, the use of scientific concepts in the formation and development of junior schoolchildren's didactic opportunities for lessons of the integrated course "I explore the world" has not been studied.

The article aim is systematization of experience in the formation and development of scientific concepts in junior schoolchildren; disclosure of didactic possibilities of the integrated course "I explore the world" in the process of formation and development of scientific concepts in junior schoolchildren (on a specific example).

World cognition around a person is one of the most important worldview problems. Cognition is "the process of purposeful active reflection of the objective world in people's minds" [20, p. 261].

Levels of cognition are:

- sensory (empirical) cognition, within which, interacting with certain objects, people receive primary knowledge about the world in sensory cognition forms, such as: sensations, perceptions, ideas, which are the result of object and subject interaction; 
- abstract (rational) cognition, forms of which are the concept, judgment, inference, by which the results of sensory data are recorded and processed in the second stage of cognition.

Human world cognition begins with the sensory reflection of reality in such forms as sensation, perception, imagination [28].

Sensation is "elementary mental process, which consists of reflecting certain properties of objects and reality phenomena, as well as the state of internal organs under the direct influence of stimuli (sound, visual, mechanical, taste, olfactory) on the senses" [23, p. 180]. In the brain there is a reflection of certain properties, external aspects of objects, phenomena that directly affect the senses. From sensations arises perception, in which the pupil already reflects the set of properties characteristic of the object, "builds" the sensory-visual image. Perception already reflects the object as a whole, in conjunction of its features [18].

Perception is "the mental process of reflection of objects, phenomena, events in general in the human brain, in the aggregate of all their qualities and properties under the direct action of stimuli on the analyzers" [23, p. 185]. A characteristic feature of perception is the naming of objects or phenomena that are perceived, i.e. inclusion of the second signal system in the perception process. It is thanks to verbal notation that people so easily and instantly perceive objects, recognize and understand them [29].

The result of sensory perception is imagination.

Imagination is "sensory images that arise on the basis of recollection of previously perceived objects (phenomena) or productive imagination" [22, p. 36].

Since representations of an object arise in the absence of the object itself, they are usually less vivid and detailed than perceptions, but more schematic and generalized: they reflect the most characteristic visual features inherent in the class of such objects. Thus, representations are not just visual images of reality, they are always to some extent generalized images of reality. Representation is always the result of individual perceptions generalization [29]. 
Further development of cognition forms that go beyond direct sensuality, makes it possible to achieve a qualitatively new level of reality reflection - rational cognition. The form of rational cognition is abstract thinking [28].

Abstract thinking is one of the types of human thinking, which consists of the formation of abstract concepts and their operation [15].

Concept is a thought expressed in a word / symbol that reflects the general, essential and necessary features in the objects of cognition [21, c. 12]. The concept is the result of the separation of general and essential features of objects or phenomena through understanding and generalization. For example, a child already has an idea of birch, linden, oak, etc. Thinking distinguishes their essential common features: one thick stem, trunk; crown of branches and leaves; plant is tall. Thus the concept of the tree is formed. As we can see, this process is already detached from sensory perception and has taken place at the level of abstract thinking.

There are two types of concepts depending on the form of objects cognition:

1. Empirical (life) concepts are pre-scientific concepts that reflect sensory features, properties. Among these signs can be significant and accidental [26].

2. Scientific concept is a form of thinking that reflects the objectively true in objects or phenomena [26].

The concept performs the following functions:

1) the discovery of new aspects of the recognizable object in the full disclosure of its essential properties [16];

2) communicative. Concepts are used as a communication means. By consolidating their knowledge in the form of concepts, people have the opportunity to share them in communication [21];

3) cognitive. The concepts that have been the result of the previous cognition process are the basis, the means of further cognition. Any concept is always revealed through a system of other concepts, and through a system of concepts of the highest generalization level [16]. 
Concepts system is knowledge of any science as a reflection of a certain part of reality, which is the subject of its knowledge.

In the context of the stated study, we consider it necessary to specify the definitions of "formation" and "development".

Formation is a process in which for some reason stability, completeness, a certain type or something is created, organized, composed, combined [30].

Development is seen by science as a process of natural change of something, the transition from one qualitative state to another - new, more perfect [27, p. 631].

Before considering the processes of formation and development of scientific concepts in junior schoolchildren, it is worth focusing on the process of forming ideas in recent years.

The idea of various objects and world phenomena is a necessary basis for mental processes. They do not arise instantly and not in the finished form, but are formed, gradually improved, changed under the influence of new, purposeful acts of perception.

Ensuring the correct perceptions formation contributes to:

1) taking into account the already existing ideas on this issue, identifying their correctness or erroneousness;

2) organization of children's communication with objects and phenomena of nature / society (direct observations, experiments performed by children independently in extracurricular activities or in lessons during work with natural objects; sometimes a natural object can be replaced by a visual aid. Methods should be accompanied by special tasks aimed at clarifying perception);

3 ) creating an image in words, when it is not possible to organize work with natural objects or their images. Such perceptions are created by the power of the creative imagination. It is useful to rely on existing perceptions and ideas [17].

It is clear that the higher the uncertainty of cognition, the more active the imagination is. But the more carefully a person studies an object or phenomenon of the world around him with the involvement of as many analyzers as possible, the less room is left for the imagination, which seems to replace knowledge and complements it. Therefore, in order for pupils to have a correct idea of the subject, phenomenon, 
process, or natural situation, the teacher must create conditions for them to irritate the receptors of as many analyzers as possible.

When organizing the process of sensory cognition of the world around, the teacher should not rely on the fact that pupils only need to provide a subject, a phenomenon to study, believing that the process of sensory cognition will occur automatically, without any help and guidance from the teacher. The process of sensory cognition is not a mechanical reflection of reality.

The effectiveness of a particular type of communication with the objects of the world is enhanced by:

- creating a problem situation with the help of correctly asked questions and tasks;

- setting a clear goal of activities and communication for children;

- asking consistent questions that direct children's attention to certain features, object properties and make them watch and listen (their wording should be short, specific, simple, but not prompted by the answer);

- organization of subjects' comparison in order to distinguish common and distinctive features;

- mastering the terms that denote the subject or its individual properties;

- creating sketches from memory, etc. [25, p. 103-104].

To form an idea, it is necessary to create the following conditions:

1) the organization of purposeful feeling and perception of signs, objects properties, and their external connections;

2) combination of sensory perception essence with verbal definition (naming of objects, phenomena, etc.);

3) the organization of the idea content awareness;

4) organization of sensory-visual image memorization of an object or group of objects;

5) the organization of the formed representation consolidation through reproduction of its maintenance without object existence; 
6) the organization of the formed representation application at the problems decision on a sample, in similar and new situations.

Thus, ideas arise on the basis of sensations and perception as a result of awareness, memorization and reproduction of objects and phenomena and become the basis for the formation of primary scientific concepts, simple in content, which are then combined into complex and more meaningful ones.

Since it is important to perceive the objects of study directly, it is necessary to involve the junior schoolchild in active, cognitive, research activities through observation, examination, experiment, practical work, measurement, systematization, classification, critical evaluation of what is seen (heard), etc. These activities form the basis of the integrated course "I explore the world" study.

Integrated course "I explore the world" is a subject aimed at forming a holistic world view in junior schoolchildren through the development of natural, civic and historical, social and health, technological, information, mathematical, linguistic and literary educational areas of the State Standard of Primary Education.

The content of the integrated course "I explore the world" covers a certain system of ideas and basic scientific concepts, which are defined in the Standard educational programs, and adapted to the age characteristics of the primary school children's mental development and general learning goals. This course involves the development of junior schoolchildren's knowledge of: objects and phenomena in nature and society; relationships and dependencies in nature and society; different types of educational and cognitive activities; special methods of objects cognition in nature and society; ways of practical activity with natural / social and man-made objects; ways of valeological and ecological behavior; general ways of educational and cognitive activities self-management; norms of ethical, aesthetic, moral attitude to objects of nature and society; norms of pupil's communication and behavior in the process of joint activities with classmates and the teacher.

The integrated course "I explore the world" in primary school involves the formation of primary concepts that bring pupils to understand the world laws for the first time. Each concept must be characterized by such a number of essential features 
that would be sufficient to interpret as primary ones. However, it must have the amount of knowledge necessary for further development.

The effectiveness of pupils' concepts mastery is reflected in two forms: concepts knowledge (not limited to definitions knowledge) and their correct operation in specific mental activity types (if pupils of $1^{\text {st }}-2^{\text {nd }}$ grades allocate, first of all, the most obvious external signs characterizing object action or its value, then in $3^{\text {rd }}-4^{\text {th }}$ grades pupils should be able to establish concepts hierarchy, to find connection between generic and specific concepts).

However, it should be noted that these forms of learning scientific concepts are often in different relationships to each other, namely:

$\checkmark$ on the one hand - the concept is mastered, but it is difficult or impossible for the pupil to operate with it, and he uses it incorrectly. This is manifested primarily when it is necessary to perform other (not similar) types of tasks;

$\checkmark$ on the other hand - the pupil is able to operate with the concept, but does not sufficiently understand its essence, i.e. cannot identify the essential features of its content.

Conscious concepts operation requires them not to be formally mastered (terms or definitions memorization), but meaningful during a specially organized cognitive activity.

Thus, the concepts formation is a specially organized, complex, holistic psychological and pedagogical process. In pedagogical practice, its components are closely intertwined. However, they perform their function in ensuring that pupils learn the concept at certain stages of this process. Each of them is dominated by a certain component: perception, awareness, comprehension, among the elements of which are generalization and systematization.

Integrating the results of psychological and pedagogical research of S. Vasiliev, P. Halperin, V. Davydov， G. Kovalyova， O. Savchenko, G. Uvarova, A. Usova, M. Shardakov and other scientists, we can distinguish five stages of scientific concepts formation process:

1. Motivational and semantic preparation for concept mastering. 
2. The organization of sensory perception of signs, objects or phenomena properties, the ideas formation or previously formed ideas actualization.

3. Mental activity organization aimed at highlighting the essential features in the cognition objects.

4. Generalization and verbal definition of the concept essence, marking it with the appropriate term.

5. Formed concept introduction in the system of available knowledge.

Specifically, we will focus on the theoretical justification and practical implementation of each of the selected stages on the example of the concept of "water cycle in nature" formation in the $2^{\text {nd }}$ grade.

First stage. Motivational and semantic preparation for concept mastering.

In order for pupils to get involved in the work during a specially organized process of concept formation, they should realize:

1) why they need to know the essence of the concept being formed;

2) what needs to be done to learn this concept consciously, i.e. to find out the motive for their activities.

The task of the first stage is to provide pupils' constant motivation to the concept formation process.

Pupils' cognitive interest and positive attitude to master the concept is provided by:

- selection of accessible, interesting material, in particular, reading excerpts from stories, fairy tales;

- demonstration of video fragments, photos, natural objects, guessing riddles, puzzles, crossword puzzles, conducting experiments, etc.

The first stage of the concept of "water cycle in nature" formation can begin with listening to the fairy tale "Dewdrop" by V. Sukhomlynskiy.

Early in the morning a drop of dew woke up on a rose.

- How did I get here? - Drop thought. - In the evening I was high in the sky. How did I get to earth?

And she wanted to go back to the sky again. 
The sun warmed up. The Drop evaporated, went high into the blue sky, to the Sun. And there were thousands of other drops! They gathered in a dark cloud and replaced the Sun.

- Why did you covered me from people? - the Sun said angrily. And sent an arrow of fire into the dark cloud. A fiery arrow struck, thunder rumbled. The dark cloud was frightened and scattered. It rained. A drop fell to the Earth.

- Thank you, Drop, - said the Earth. - I missed you so much!

After listening to the fairy tale, the teacher asks the children:

- Where did the Drop get on the rose in the morning and how was she able to rise high in the sky to the Sun?

At this stage, the teacher also reveals pupils' basic knowledge, which is the basis for the new concept formation (What types of precipitation do you know? Where does water disappear from puddles? What is water vapor? What is evaporation? How does sunlight affect water-liquid?).

It is important to find out whether pupils have realized the essence of the basic concepts, whether they know how to operate in any situation.

The second stage. Sensory perception organization of signs, objects or phenomena properties, ideas formation about them or actualization of previously formed ideas.

Theoretical assimilation of scientific concepts is impossible without the child's sensory experience, which consists of objects and phenomena of nature and society that are perceived. Perception occurs through the sensation of their individual characteristics (size, color, shape, weight, place in space and time, etc.), which act on the subject analyzers. All these features do not exist themselves, but belong to a specific object. Therefore, in the child's mind there is his image.

The task of this stage is to ensure that pupils consciously perceive objects and phenomena of nature / society.

The conscious perception of environmental objects and phenomena is influenced by properly organized observation in nature, observation of natural objects, experiments, organization of independent educational search, use of various teaching 
aids, previously acquired knowledge and practical experience, accurate and figurative teacher's words, exercises that clarify perceptions.

At this stage, the teacher can offer an experiment demonstration.

Experiment. On a tripod one under another the teacher puts: alcohol, a vessel with water, a plate with ice. Pupils watch as the steam from the boiling water in the vessel rises, touching the cold plate from below, turns into water, and the ice in the plate begins to melt and turn into water. That is, there is an observation of water evaporation and steam condensation.

The teacher conducts a conversation (based on what has been seen):

- What do you see in the vessel?

- Is there anything in common between a vessel with water, which we heat, and a plate with ice?

- Where did the water droplets come from?

- What happened to them?

At this stage, the educator teaches children to distinguish objects' features and properties.

The third stage. Mental activity organization aimed at highlighting the essential features in objects of cognition.

Mental activity begins when the pupil has a sufficient number of facts, ideas, i.e. material for thinking, in his mind obtained at the previous stage. In scientific concept formation the understanding of material plays an important role, which in the process of assimilation is carried out through the analysis of perceived features of objects or phenomena of the world, comparison, abstraction and logical sorting of their essential and insignificant features through mental transition from individual features or phenomena to the essential ones, the establishment of interdependencies and relationships between them, as well as the generalization of essential features.

The task of this stage is to identify the essential features in the objects of cognition, to establish relationships and interdependencies between them. 
To implement these tasks, pupils need to have the techniques of mental activity (analysis, synthesis, abstraction, generalization, systematization, etc.), the ability to reason, express their own opinions, to prove their objectivity.

At this stage, it is appropriate to conduct a research workshop.

Research workshop. Where does the water from the puddles disappear?

- After the rain, the streets and roofs of houses are wet, there are puddles everywhere. But the sun will come up and everything will dry up. Where does the water go? (It evaporates, turns into an invisible vapor). What happens to water in rivers, lakes, seas? (It also evaporates).

- Where does water evaporate faster: from a plate or a glass? (Water evaporates faster from the plate. The larger the surface of the water, the faster it evaporates).

- Does water evaporate in cold weather? (It also evaporates, but not as fast as in the heat).

The fourth stage. Generalization and verbal definition of the concept essence, marking it with the appropriate term.

In the process of understanding the essential concept features there is a conceptual (local) generalization, which leads to the individual concepts formation. The concept meaning is summarized in the definition.

The task of this stage is to generalize the cognition objects features singled out at the previous stage in the form of an accessible definition and mark it with the appropriate term.

At this stage, based on what he saw and as the analysis result, the teacher introduces a new concept of "water cycle in nature."

- With the help of experiments, we observed the transformation of water-liquid into water vapor, ice into water-liquid. And how in nature are the transformations of one state into another?

- On Earth, under the sunlight, water constantly evaporates from the surface of rivers, lakes, seas, oceans, moist soil, plant leaves, animal and human bodies. When invisible steam cools, it forms very small droplets of water that collect in the clouds. Small drops in the clouds, merging with each other, gradually increase. Heavy drops 
of water cannot stay in the air, so they fall to the ground in summer as rain, and in winter as snow.

- Returning to land in the form of precipitation, water flows down the slopes and collects in streams and rivers flowing into lakes, seas and oceans. Part of the water seeps through the soil and rocks, reaches groundwater, which also flows into rivers or other water bodies.

- Thus, the continuous process of moving water from the oceans to land through the air and from land back to the ocean is called the water cycle in nature.

The fifth stage. Introduction of the formed concept into the system of available knowledge.

At this stage it is necessary to make generalizations. It consists of uniting the learned concepts into a system, in revealing connections and relations between the elements of this system, in arranging them in a certain order and rational sequence. Pupils learn to consider and apply the concept of "water cycle in nature".

- Look at the picture and analyze it. How does the water cycle in nature?

- Why is there less water in rivers, ponds and lakes in summer?

- At what times of the year is there water transformation in nature from liquid to gaseous? From solid to liquid?

- How do you understand the phrase: "Water is an eternal traveler"?

The children express their ideas, and the teacher writes them on the board. The collected ideas are grouped, analyzed, and those that answer the question are selected. A concept is considered formed if pupils are free to operate with it in different educational and life situations.

Each concept at a certain stage of the learning process has its initial and final formation level, contained in the program and textbook. It is introduced gradually, in several stages. The concepts formation in the learning process of the integrated course "I explore the world" should be carried out in different ways. Their choice depends on the concept content, children's general development level, their cognitive abilities and the basic knowledge and skills availability. 
It should be noted that individual concepts are formed on the basis of ideas, and general ones - on the basis of concepts that are characterized by a smaller volume.

The concept acquires an evidential and convincing character if its essential features are confirmed by the optimal number of facts and if its interrelations with other concepts are considered. This indicates the importance of building a logically connected concepts system. Therefore, an important feature of the integrated course "I explore the world" is the systematic concepts introduction, which involves the connection of each new concept with the already studied one.

The formation of scientific concepts is a long process because pupils continue working with the same objects in different parts of the course. There is a gradual concept development by expanding its scope and deepening its meaning.

In its development, the concept goes through three stages.

The first stage of concept development is characterized by the fact that its essential features are still based on sensory experience. They are abstracted on the basis of direct perception of objects and phenomena or their images and contain a fairly small number of knowledge elements, a low degree of generalization. These are elementary or primary concepts.

In the second stage of development, the concept is characterized by a higher degree of abstraction. Its essential features are hidden from "living contemplation" and are a generalization of elementary concepts characteristics. Such concepts can be concretized indirectly, through a number of simple concepts.

The third stage of concept development is characterized by the highest degree of generalization, abstraction, when the concept acquires the status of regularity or theory. The degree of its distance from the sensory expeience is so great that it gives the impression of its non-involvement in this experience and is often seen as the result of pure abstraction.

Concepts can remain at the elementary level, and the whole stock of child's knowledge will be a set of disparate, chaotic truths, if this process is not controlled by the teacher. 
The knowledge repetition, the revival of existing sensory experience are important in the development of concepts. Repetition, which is carried out not immediately after the first material perception, but after some time, after 2-3 days, is extremely effective for the concepts development. It is important that the repetition takes place in the form of in-depth understanding and even rethinking of the studied material, the establishment of new associative connections.

As a rule, enrichment with new characteristics of already existing concepts is at the stage of new knowledge formation. So that the new characteristics do not turn out to be isolated, the teacher must refer to the previously studied material and establish logical connections with at the stage of learning new material. Sometimes it is necessary to refer to the educational material of previous years. For example, mastering the concept of "Sun is the central body of the solar system" in 4th grade requires the knowledge reproduction about the importance of the Sun for life on Earth, the influence of the Sun on seasonal phenomena in nature, the Sun as an energy source on Earth, which are obtained in 1st, 2nd and 3rd grades. This, in turn, will enrich the above concept, and previously studied will not just be mechanically reproduced, but included in a certain knowledge system at another, higher level. Meanwhile, the assimilation of each new concept characteristic goes through the same stages as the original concept formation. But now this new feature is linked to the already known ones.

Deeper understanding and development of concepts is facilitated by various exercises and practical work, where knowledge is tested in practice. The concepts development is accompanied by an increase in pupils' vocabulary, as the concepts are fixed in terms, symbols, definitions, which are their material carriers.

Thus, the concepts formation and development is a long process in which the concept is enriched with new characteristics with the constant acquisition of new knowledge, their understanding and connection with existing knowledge. The management of this process is carried out by the teacher through: repetition system and practical knowledge mastering; increasingly complex issues application; tasks to establish logical connections between individual learned concepts. In this process, the integrated course "I explore the world" is extremely important, as it involves the 
formation of ideas and concepts about the world integrity; natural and social environment as an environment of human life, its belonging to nature and society; empirical and generalized ideas and concepts that reflect the basic properties and patterns of the real world, expand and organize pupils' social and cognitive experience.

The study does not claim to be a comprehensive consideration of all aspects of this problem. The factors of junior schoolchildren's scientific concepts formation, as well as the selection and use of technologies, methods, teaching aids in the lessons of the integrated course "I explore the world" in the context of scientific concepts formation and development in junior schoolchildren need further study. 